\title{
S-GRAS score for prognostic classification of adrenocortical carcinoma: an international, multicenter ENSAT study
}

\author{
Y S Elhassan (10,2, B Altieri ${ }^{3}$, S Berhane ${ }^{4,5}$, D Cosentini ${ }^{6}$, A Calabrese ${ }^{7}$, M Haissaguerre ${ }^{8}$, D Kastelan 9 , \\ M C B V Fragoso ${ }^{10}$, J Bertherat ${ }^{11}$, A Al Ghuzlan ${ }^{12}$, H Haak ${ }^{13}$, M Boudina ${ }^{14}$, L Canu(15, P Loli ${ }^{16}$, M Sherlock ${ }^{17}$, \\ O Kimpel ${ }^{3}$, M Laganà ${ }^{6}$, A J Sitch ${ }^{4,5}$, M Kroiss $\mathbb{1 D}^{3,18,19}$, W Arlt ${ }^{1,2,4}$, M Terzolo7, A Berruti ${ }^{6}$, J J Deeks ${ }^{4,5}$, R Libé20, \\ M Fassnacht $\mathbb{1}^{3,18}$ and $C$ L Ronchi ${ }^{10,2,3}$ on behalf of the ENSAT
}

${ }^{1}$ Institute of Metabolism and Systems Research, University of Birmingham, Birmingham, UK, ${ }^{2}$ Department of Endocrinology, Queen Elizabeth Hospital, University Hospitals Birmingham NHS Foundation Trust, Birmingham, UK, ${ }^{3}$ Division of Endocrinology and Diabetes, Department of Internal Medicine I, University Hospital, University of Würzburg, Würzburg, Germany, ${ }^{4}$ NIHR Birmingham Biomedical Research Centre, University Hospitals Birmingham NHS Foundation Trust and University of Birmingham, Birmingham, UK, ${ }^{5}$ nstitute of Applied Health Research, University of Birmingham, Birmingham, UK, ${ }^{6}$ Medical Oncology, Department of Medical and Surgical Specialties, Radiological Sciences and Public Health University of Brescia, ASST-Spedali Civili, Brescia, Italy, ${ }^{7}$ Department of Clinical and Biological Sciences, University of Turin, San Luigi Hospital, Orbassano, Italy, ${ }^{8}$ Service d'Endocrinologie - Diabète et Nutrition CHU de Bordeaux, Bordeaux, France, ${ }^{9}$ Department of Endocrinology, University Hospital Centre Zagreb, Zagreb, Croatia, ${ }^{10}$ Unidade de Suprarrenal da Disciplina de Endocrinologia e Metabologia da Faculdade de Medicina do Hospital das Clinicas da Universidade de São Paulo (HCFMUSP), and Instituto do Cancer do Estado de Sao Paulo (ICESP), Sao Paulo, Brazil, ${ }^{11}$ Reference Center for Rare Adrenal Cancer (COMETE), Cochin Hospital, Paris, France, ${ }^{12}$ Department of Pathology, Gustave Roussy Cancer Center, Paris, France, ${ }^{13}$ Department of Internal Medicine, Máxima MC, Eindhoven, Netherlands, ${ }^{14}$ Department of Endocrinology, Theagenio Cancer Hospital, Thessaloniki, Greece, ${ }^{15}$ Department of Experimental and Clinical Biomedical Sciences, University of Florence, Florence, Italy, ${ }^{16} \mathrm{Clinica}$ Polispecialistica San Carlo, Paderno Dugnano, Milano, Italy, ${ }^{17}$ Department of Endocrinology, Beaumont Hospital, and the Royal College of Surgeons, Dublin, Republic of Ireland, ${ }^{18}$ Comprehensive Cancer Center Mainfranken, University of Würzburg, Würzburg, Germany, ${ }^{19}$ Department for Endocrinology, Medizinische Klinik und Poliklinik IV, Ludwig-Maximilians-University, Munich, Germany, and ${ }^{20}$ Department of Endocrinology and Metabolic Diseases, Hôpital Cochin, Paris, France

Correspondence should be addressed to C L Ronchi Email

C.L.Ronchi@bham.ac.uk

\section{Abstract}

Objective: Adrenocortical carcinoma (ACC) has an aggressive but variable clinical course. Prognostic stratification based on the European Network for the Study of Adrenal Tumours stage and Ki67 index is limited. We aimed to demonstrate the prognostic role of a points-based score (S-GRAS) in a large cohort of patients with ACC.

Design: This is a multicentre, retrospective study on ACC patients who underwent adrenalectomy.

Methods: The S-GRAS score was calculated as a sum of the following points: tumour stage $(1-2=0 ; 3=1 ; 4=2)$, grade (Ki67 index 0-9\%=0;10-19\%=1; $\geq 20 \%=2$ points), resection status ( $R 0=0 ; R X=1 ; R 1=2 ; R 2=3)$, age $(<50$ years $=0$; $\geq 50$ years $=1$ ), symptoms (no $=0$; yes $=1)$, and categorised, generating four groups $(0-1,2-3,4-5$, and $6-9)$. Endpoints were progression-free survival (PFS) and disease-specific survival (DSS). The discriminative performance of S-GRAS and its components was tested by Harrell's Concordance index (C-index) and Royston-Sauerbrei's $\mathrm{R}_{\mathrm{D}}{ }_{\mathrm{D}}$ statistic.

Results: We included 942 ACC patients. The S-GRAS score showed superior prognostic performance for both PFS and DSS, with best discrimination obtained using the individual scores $(0-9)\left(C\right.$-index $=0.73, R_{D}^{2}=0.30$, and C-index $=0.79, R_{D}^{2}=$ 0.45 , respectively, all $P<0.01$ vs each component). The superiority of S-GRAS score remained when comparing patients treated or not with adjuvant mitotane ( $n=481 \mathrm{vs} 314$ ). In particular, the risk of recurrence was significantly reduced as a result of adjuvant mitotane only in patients with S-GRAS 4-5.

Conclusion: The prognostic performance of S-GRAS is superior to tumour stage and Ki67 in operated ACC patients, independently from adjuvant mitotane. S-GRAS score provides a new important guide for personalised management of ACC (i.e. radiological surveillance and adjuvant treatment). Printed in Great Britain

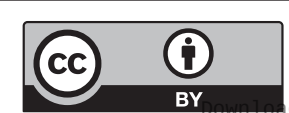

This work is licensed under a Creative Commons Attribution 4.0 International License. 


\section{Introduction}

Adrenocortical carcinoma (ACC) is a rare malignancy with an incidence of $0.7-2 /$ million/year $(1,2)$. Although prognosis in ACC is generally unfavourable, there is wide heterogeneity in the outcomes. The 5-year survival ranges from 10 to $60 \%$, mostly reflecting the primary tumour stage and the resection status $(3,4)$. However, up to $60 \%$ of patients with localised tumours experience disease recurrence after radical surgery (5) and $15 \%$ die within 2 years (3). Reliable prognostication after ACC resection is critical to guide frequency of follow-up, adjuvant treatment, and to more accurately counsel patients regarding long-term outcomes.

Nonetheless, the optimal tools for prognostication in ACC are debatable. Despite the considerable heterogeneity, the European Network for the Study of Adrenal Tumours (ENSAT) staging system is widely used as the standard prognostic factor in $\operatorname{ACC}(3,6$, 7). To better prognosticate patients with advanced disease, a modified ENSAT stage has been proposed which sub-classifies patients to account for involved regional lymph nodes and number of metastatic organs (mENSAT stages 4a, 4b, and 4c) (8). Among other prognostic characteristics, the Ki67 proliferation index (9) is considered the most important single parameter $(6,10,11)$. Incomplete tumour resection is also linked to unfavourable prognosis $(12,13)$. Finally, although older age and steroid excess have been associated with decreased survival $(14,15,16)$, their prognostic value remains uncertain $(17,18)$.

Due to the shortcomings of individual clinical/ histopathological prognostic markers, combined scores were studied for better prognostic accuracy in ACC. We firstly demonstrated the prognostic value of the GRAS components, that is, grading ( $G$, Weiss score $>6$ and/or Ki67 $\geq 20 \%$ ), resection status (R), age (A) and tumour- or hormone-related symptoms (S)) in 444 patients with advanced ACC (8). More recently, in 107 patients, we reported that a modified form of the GRAS classification, here termed S-GRAS, which includes the ENSAT stage and focuses on Ki67 for grading, allows better stratification than individual clinical/histopathological characteristics (19).

Herein, we aimed to demonstrate the prognostic performance of the S-GRAS score in the largest study cohort of well-characterised ACC patients to-date. We also investigated the S-GRAS prognostic role in relation to adjuvant mitotane treatment, being the most utilised adjuvant therapy in ACC $(20,21)$.

\section{Methods}

\section{Patients and data collection}

This is a retrospective, international, multicentre study conducted on behalf of ENSAT (www.ensat.org). Details of the recruitment process and data collection are reported in Supplementary Table 1 (see section on supplementary materials given at the end of this article). The inclusion criteria were: (i) age $\geq 18$ years, (ii) histologically confirmed ACC, (iii) available clinical/histopathological characteristics at diagnosis to allow the calculation of the S-GRAS score, and (iv) available follow-up radiological data to determine disease status and survival. We excluded non-operated ACC patients and those included in our two previous studies (Libé et al. (8) and Lippert et al. (19)). Given the exclusion of patients with inoperable disease, we did not consider the previously proposed mENSAT classification for patients with advanced ACC (8).

Mandatory data included: sex, age at diagnosis, presence of tumour- or hormone-related symptoms at presentation, adrenal hormone status, ENSAT stage, date of adrenalectomy, resection status $(\mathrm{R} 0=$ complete resection, $\mathrm{RX}=$ cannot be assessed, $\mathrm{R} 1=$ indicates the removal of all macroscopic disease, but microscopic margins are positive for tumour, or $\mathrm{R} 2=$ indicates gross residual disease with post-operative residual disease that was not resected (primary tumour, regional nodes, and macroscopic margin involvement), Ki67 index, date of disease recurrence or progression after primary surgery, and date of last follow-up or death. Symptoms were defined as: hormone-related if due to adrenal hormone excess (e.g. glucocorticoids - Cushing syndrome; androgens - hirsutism, acne, and alopecia; mineralocorticoids uncontrolled blood hypertension), tumour-related if due to mass effect (e.g. abdominal pain), or systemic cancerrelated (e.g. fatigue or weight loss). Patients with ENSAT stage 4 with complete resection of primary tumours and metastases were considered R0. Patients with ENSAT stage 4 with residual disease manifestations were defined as R2.

We calculated the S-GRAS score as previously described (19): age at diagnosis $(<50$ years $=0$ point; $\geq 50$ years $=1$ point), hormone, tumour or systemic cancer-related symptoms at presentation (no $=0$ point; yes $=1$ point), ENSAT stage ( 1 or $2=0$ point; $3=1$ point; $4=2$ points), $\mathrm{R}$ of primary tumour ( $\mathrm{R} 0=0$ point; $\mathrm{RX}=1$ point; $\mathrm{R} 1=2$ points; $\mathrm{R} 2=3$ points), and Ki67 index $(0-9 \%=0$ point; $10-19 \%=1$ point; $\geq 20 \%=2$ points) (Supplementary Table 2), generating ten S-GRAS scores and four S-GRAS groups: 0-1, 2-3, 4-5, and 6-9 (19). Given the exclusion of 
patients with inoperable disease, we did not consider the previously proposed mENSAT classification for patients with advanced ACC (8).

Disease monitoring was done through periodical cross-sectional imaging as detailed in Supplementary Table 1. We also collected details of whether adjuvant treatment with mitotane (o,p'-DDD) was used after radical surgery or not (21). Modalities of initiation and titration of mitotane treatment are reported in Supplementary Table 1.

All participating institutions obtained local ethics approval for recording of pseudonymised and standardised data in the ENSAT registry for use in any current and future adrenal tumour-related projects (www.ensat. org). The entire list of the participating centres as well as the details of the ethical approval are provided in the Supplementary Table 1. All participants provided written informed consent.

\section{Endpoints}

Primary outcomes were: (i) progression-free survival (PFS) defined as the time from primary tumour resection to the first radiological evidence of progression (e.g. disease relapse in patients after radical resection or progressive and/or new lesions in patients with advanced disease, i.e. $\mathrm{R} 2$, as defined by local radiologists) and (ii) disease-specific survival (DSS) defined as the time from primary resection of ACC to disease-related death.

DSS was chosen against overall survival to have a more accurate measure of the clinical prognosis, avoiding a potential overestimation of mortality (especially of older patients).

\section{Statistical analysis}

Statistical analysis was undertaken using Stata/SE Version 16.0 (StataCorp).

Post hoc power calculation was based on the method described by Jinks et al. (22) (Stata command dsampsi) that relies on a target value of the Royston and Sauerbrei D measure of discrimination (22). Details are reported in the Supplementary Table 3. With 942 patients in this study, we have adequate sample size for the evaluation of S-GRAS to cover a range of possible values of $\mathrm{D}$.

Continuous variables were presented as median and interquartile range and categorical variables as counts and percentages. For each of the DSS and PFS endpoints, we performed Kaplan-Meier (KM) survival curves according to the S-GRAS score, ENSAT stage, Ki67 index, resection status, age, and symptoms. Median survival, percentage survival at 24 and 60 months were reported for each survival curve. The prognostic effect of S-GRAS score and its individual components was examined using univariable Cox regression. Hazard ratio (HR), 95\% CIs, and $P$-values were reported. $P$-values $<0.05$ were considered significant. The discriminative performance of the four S-GRAS groups and ten individual scores were compared to the single components using the Harrell's Concordance index (C-index) (23), and the Royston-Sauerbrei's Discrimination $\mathrm{R}^{2}{ }_{\mathrm{D}}$ statistic (24). Harrell's C-index is the proportion of
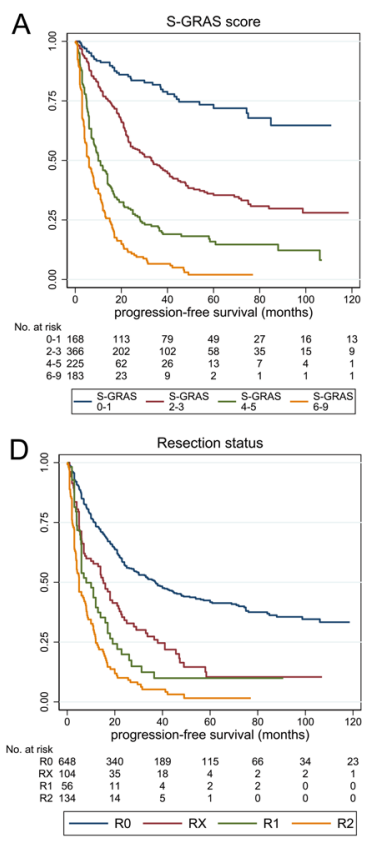
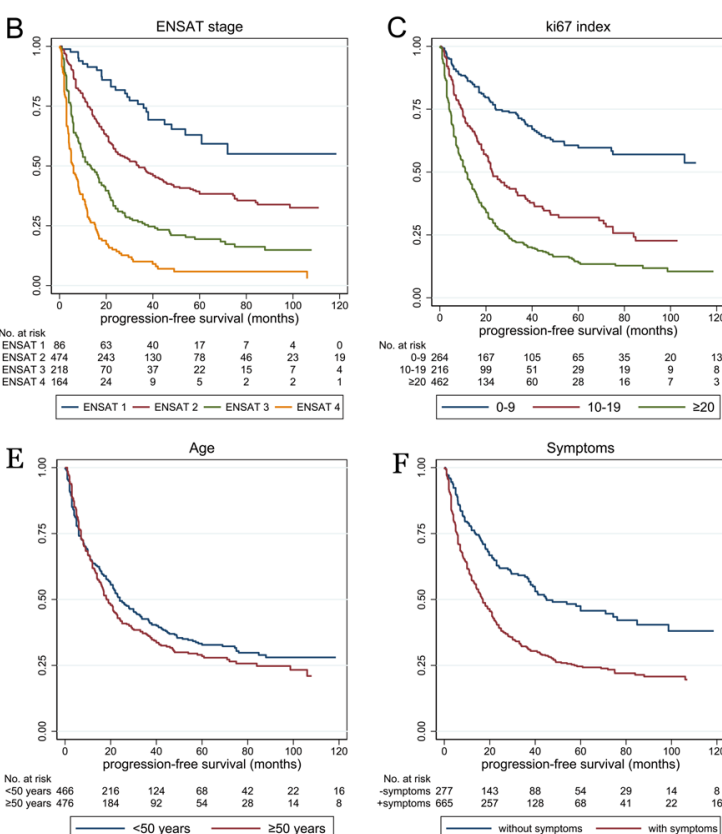

Symptoms

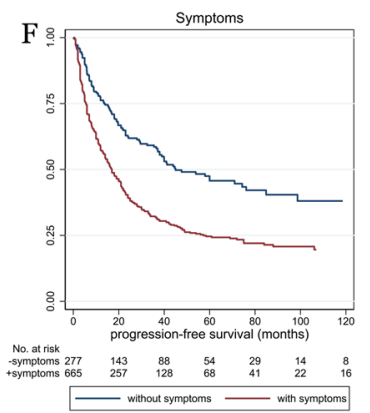

\section{Figure 1}

Kaplan-Meier curves depicting progression-free survival according to S-GRAS score grouping and each S-GRAS component $(n=942)$. (A) S-GRAS groups 0-1, 2-3, 4-5, and 6-9, (B) ENSAT tumour stage (1-4), (C) Ki67 proliferation index $(0-9,10-19, \geq 20)$, (D) resection status of primary tumour (R0, RX, R1, and R2), (E) age at time of diagnosis $(<50, \geq 50)$, (F) symptoms at time of diagnosis. For univariate statistical analysis, see Table 1. 


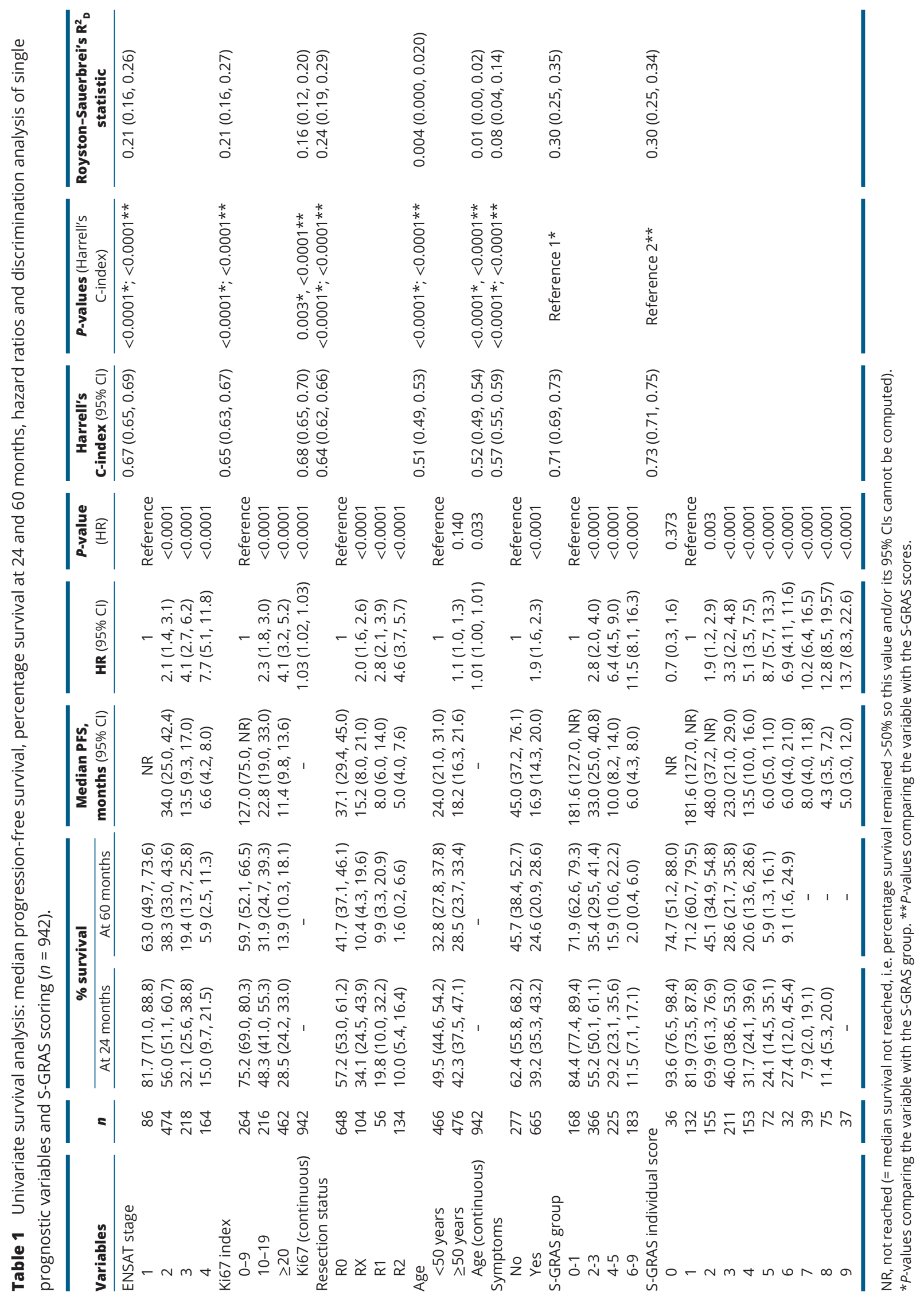


patient pairs where the predicted and observed survival outcomes are in agreement with respect to rank. $\mathrm{R}_{\mathrm{D}}^{2}$ measures the level of explained variation on the log relative hazard scale. A higher value of Harrell's C-index and $\mathrm{R}_{\mathrm{D}}^{2}$ is indicative of better model discrimination.

Finally, the added value of resection, age, and symptom status (RAS components) was evaluated by comparing multivariable Cox regression models with and without these three factors added to ENSAT tumour stage (S) and Ki67 index (G) using a likelihood ratio test.

\section{Subgroup analyses}

The performance of S-GRAS score and its components was also assessed within patients with R0 resection status for the recurrence-free survival (RFS), defined as the time from primary adrenalectomy to the first radiological evidence of relapse $(n=648)$.

Excluding patients with R2 status and those with incomplete treatment data, a subgroup analysis was performed comparing patients treated with adjuvant mitotane $(n=481)$ with those untreated $(n=314)$. Aforementioned methods were repeated, but an interaction term was introduced to the univariable Cox regressions to determine if the prognostic effect of S-GRAS score, ENSAT stage, Ki67 index, and resection status varied according to mitotane treatment status. Specifically, marginal effect statistics were derived from univariable Cox regressions that included an interaction term between each score and mitotane treatment status. These measured the change in predicted HR if status changed from no treatment to treatment. KM survival curves according to treatment status were plotted. The $\mathrm{C}$-index and $\mathrm{R}_{\mathrm{D}}^{2}$ statistic were also reported for each subgroup.

\section{Results}

\section{Patient demographics}

The 14 participating ENSAT centres identified 1075 ACC patients who underwent adrenalectomy between 2010 and 2019 (Supplementary Fig. 1). A final cohort of 942 eligible patients were enrolled. The baseline characteristics and follow-up data are summarised in Supplementary Table 4. Briefly, $62 \%$ of patients were women, $70 \%$ presented with symptoms, 50\% had ENSAT stage 2 disease, $69 \%$ had R0 status, $49 \%$ had Ki67 index $\geq 20 \%$, and most developed disease recurrence or progression during follow-up (61\%). Overall, 281 patients died of ACC (30\%), while additional 14 patients died for other reasons $(2 \%, 9$ of whom were tumour-free at the time of death).

\section{Prognostic performance of the four S-GRAS score groups}

\section{Progression-free survival}

KM curves for the S-GRAS groups and its components are shown in Fig. 1A, B, C, D, E and F. Univariable survival analysis showed all the evaluated variables
A
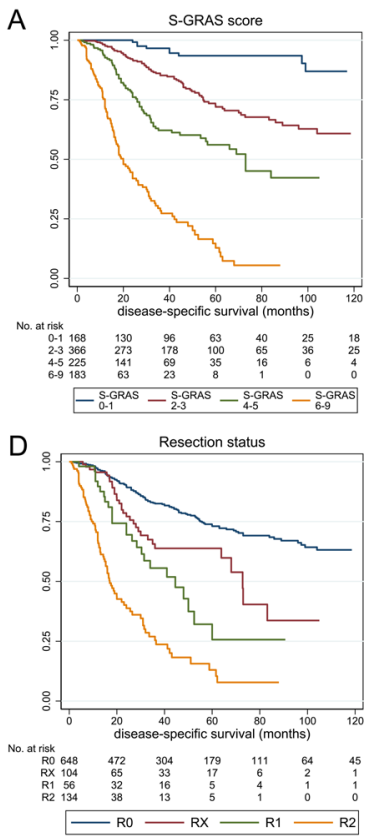
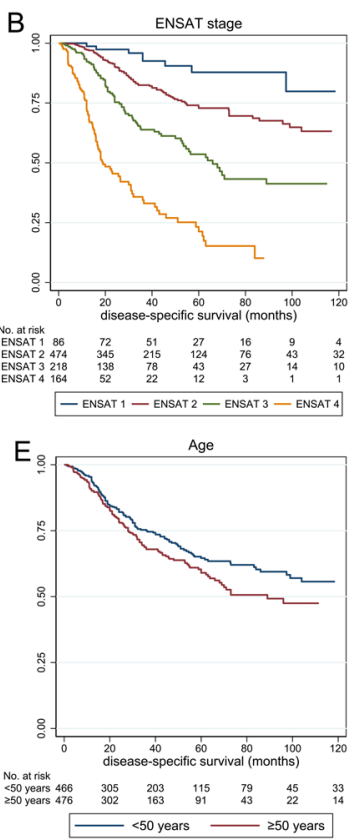
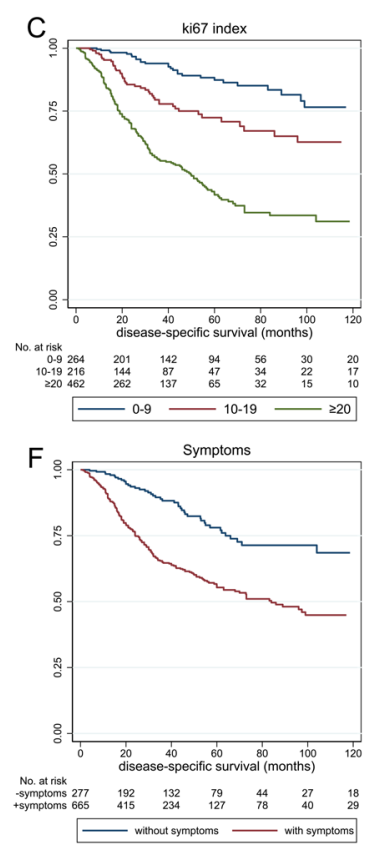

\section{Figure 2}

Kaplan-Meier curves depicting diseasespecific survival according to S-GRAS score grouping and each S-GRAS component $(n=942)$. (A) S-GRAS groups 0-1, 2-3, 4-5, and 6-9, (B) ENSAT tumour stage (1-4), (C) Ki67 proliferation index $(0-9,10-19, \geq 20)$, (D) resection status of primary tumour (R0, RX, R1, and R2), (E) age at time of diagnosis $(<50, \geq 50)$, (F) symptoms at time of diagnosis. For univariate statistical analysis, see Table 1. 
being strongly associated with PFS, except age (Table 1). The discrimination statistics showed the superiority of S-GRAS compared to all its components (Harrell's C-index $P<0.0001$ vs each component, $P=0.003$ vs continuous Ki67, Table 1). Specifically, the S-GRAS groups showed better performance (C-index $=0.71, \mathrm{R}_{\mathrm{D}}^{2}=0.30$ ) compared to ENSAT staging (C-index $=0.67, \mathrm{R}_{\mathrm{D}}^{2}=0.21$ ), and Ki67 index (C-index $=0.65, \mathrm{R}_{\mathrm{D}}^{2}=0.21$ for $0-9 \%, 10-19 \%$, and $\geq 20 \%$ grouping). There was a significantly higher risk of disease progression in patients with S-GRAS 2-3, 4-5, and 6-9 compared to S-GRAS 0-1 (2.8, 6.4, and 11.5 times, respectively, $P<0.0001$ for all, Table 1). Moreover, S-GRAS 0-1 identified a larger group of ACC patients with longer PFS ( $n=168,72 \%$ PFS at 60 months) compared to ENSAT stage 1 ( $n=86,63 \%$ PFS at 60 months).

Finally, the comparison between the multivariable Cox regression models with and without resection, age, and symptom status added to ENSAT stage and Ki67 index showed that adding the three variables resulted in a significant improvement $(P<0.0001)$ in the model fit (Supplementary Table 5A). The Harrell's C-index was also higher in the larger model (0.733 vs 0.717 ).

\section{Disease-specific survival}

As the number of ACC-unrelated deaths was small (14 out of 295 total deaths), there was no statistically significant difference between overall survival and DSS (median 104 vs 123 months, $P=0.4764$ ). Therefore, DSS was considered as an approximate of overall survival. Univariable analysis showed all variables being strongly associated with DSS (Supplementary Table 6). Again, the four S-GRAS groups performed better compared to the individual components $(P<0.0001$ for Harrell's C-index), showing higher C-index and $\mathrm{R}_{\mathrm{D}}^{2}\left(\mathrm{C}\right.$-index $\left.=0.77, \mathrm{R}_{\mathrm{D}}^{2}=0.46\right)$ than ENSAT stage (C-index $\left.=0.72, \mathrm{R}_{\mathrm{D}}^{2}=0.35\right)$ and $\mathrm{Ki} 67$ index (C-index $=0.69, \mathrm{R}_{\mathrm{D}}^{2}=0.31$ for $0-9 \%, 10-19 \%$, and $\geq 20 \%$ grouping). Compared to S-GRAS 0-1, patients with S-GRAS 2-3, 4-5, and 6-9 had 3.4, 7.8, and 27.3 times higher risk of disease-related death, respectively (Fig. 2A, B, C, D, E, and F; $P<0.0001$ for all). S-GRAS group $0-1$ patients had longer DSS ( $n=168,93 \%$ DSS at 60 months) compared to ENSAT stage 1 ( $n=86,88 \%$ DSS at 60 months, Supplementary Table 6), and S-GRAS group 6-9 showed worse clinical outcome than ENSAT4 (13 vs $21 \%$ DSS at 60 months).

As with the PFS outcome, multivariable Cox regression models showed that adding resection status, age, and symptoms to ENSAT stage and Ki67 resulted in a significant improvement in model fit $(P<0.0001)$ (Supplementary Table 5B). This led to an increase in the Harrell's C-index from 0.771 to 0.797 .

\section{Prognostic performance of the individual S-GRAS scores}

Importantly, the ten individual S-GRAS scores (0-9) further stratified subgroups of patients with different clinical outcomes for PFS and DSS. KM plots and median survival data, percentage survival at 24 and
A
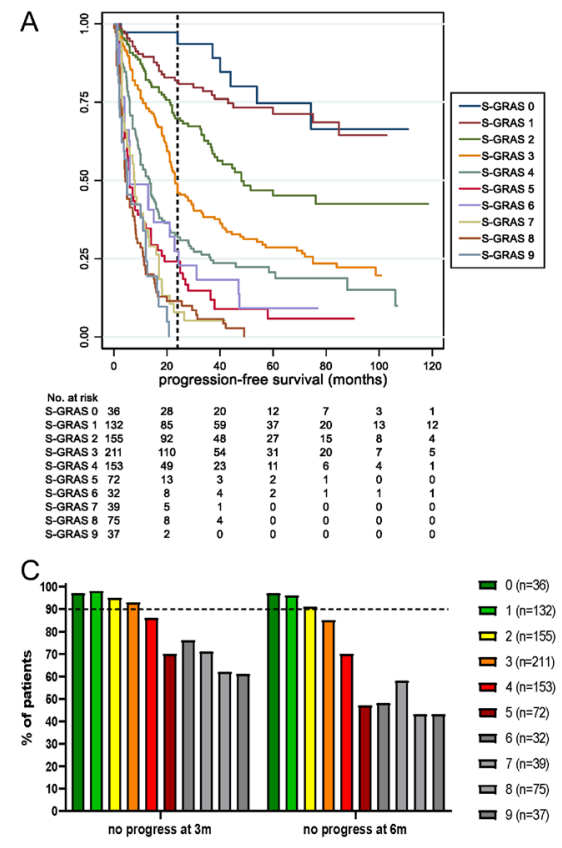
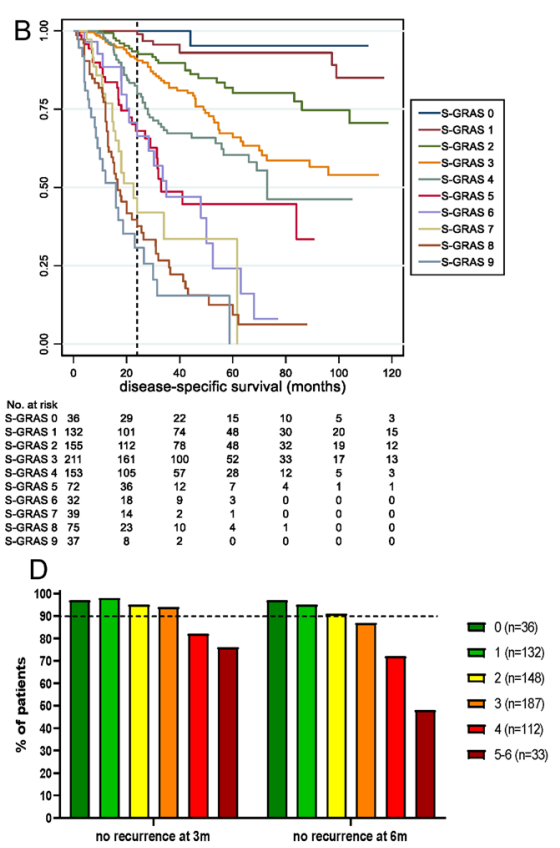

\section{Figure 3}

Prognostic role of the individual S-GRAS score categories (0-9). (A) Kaplan-Meier (KM) survival curves for progression-free survival; (B) KM survival curves for disease-specific survival. (C) Percentages of patients without documented disease progress at routine surveillance imaging after 3 and 6 months from primary surgery according to individual S-GRAS scores $(n=942)$. (D) Percentages of patients without documented disease recurrence (after complete resection, $n=$ 648 ) at routine surveillance imaging after 3 and 6 months from primary surgery according to individual S-GRAS scores. (C and D) Number of patients in each score group in brackets. Dotted line set at $90 \%$. 
Table 2 Univariate survival analysis: median recurrence-free survival (RFS), hazard ratios and discrimination analysis of single prognostic variables and S-GRAS scoring in patients with adrenocortical carcinoma treated or not treated with mitotane (MT) adjuvant $(n=795)$.

\begin{tabular}{|c|c|c|c|c|c|c|c|c|}
\hline $\begin{array}{l}\text { Variables/MT } \\
\text { status }\end{array}$ & $n$ & $\begin{array}{c}\text { Median RFS, } \\
\text { months }(95 \% \mathrm{Cl})\end{array}$ & HR $(95 \% \mathrm{Cl})$ & $\begin{array}{c}\text { P-value } \\
(H R)\end{array}$ & $\begin{array}{c}\text { HR difference } \\
\text { - untreated vs } \\
\text { treated }(95 \% \mathrm{Cl})\end{array}$ & $\begin{array}{c}\begin{array}{c}P \text {-value } \\
\text { (difference) }\end{array} \\
\end{array}$ & $\begin{array}{c}\text { Harrell's C-index } \\
(95 \% \mathrm{Cl}) \\
\end{array}$ & $\begin{array}{c}\text { Royston- } \\
\text { Sauerbrei's } \\
\mathbf{R}^{2} \text { statistic }\end{array}$ \\
\hline $\begin{array}{l}\text { ENSAT stage } \\
(\mathrm{MT}=\mathrm{No})\end{array}$ & & & & & & & $0.67(0.63,0.71)$ & $0.18(0.11,0.26)$ \\
\hline 1 & 47 & NR & 1 & Reference & & & & \\
\hline 2 & 172 & $36.5(21.0,45.6)$ & $2.6(1.5,4.7)$ & 0.001 & & & & \\
\hline 3 & 80 & $8.0(5.0,16.0)$ & $7.1(3.9,12.9)$ & $<0.0001$ & & & & \\
\hline 4 & 15 & $5.3(2.9, N R)$ & $6.4(2.9,14.3)$ & $<0.0001$ & & & & \\
\hline $\begin{array}{l}\text { ENSAT stage } \\
(\mathrm{MT}=\text { Yes })\end{array}$ & & & & & & & $0.60(0.56,0.63)$ & $0.07(0.03,0.13)$ \\
\hline 1 & 38 & $60.9(29.0, N R)$ & $1.6(0.7,3.4)$ & 0.242 & $0.58(-0.63,1.80)$ & 0.348 & & \\
\hline 2 & 291 & $34.0(26.0,48.0)$ & $2.6(1.5,4.6)$ & 0.001 & $-0.01(-0.70,0.68)$ & 0.976 & & \\
\hline 3 & 130 & $17.0(11.0,22.0)$ & $4.2(2.3,7.5)$ & $<0.0001$ & $-2.90(-5.42,-0.39)$ & 0.024 & & \\
\hline 4 & 22 & $8.0(5.7,24.4)$ & $6.3(3.0,12.9)$ & $<0.0001$ & $-0.11(-4.92,4.69)$ & 0.963 & & \\
\hline $\begin{array}{l}\text { Ki67 index } \\
\quad(\mathrm{MT}=\mathrm{No})\end{array}$ & & & & & & & $0.70(0.66,0.74)$ & $0.32(0.23,0.41)$ \\
\hline $0-9$ & 141 & 199.8 (NR, NR) & 1 & Reference & & & & \\
\hline $10-19$ & 68 & $21.0(15.2,36.9)$ & $2.9(1.9,4.5)$ & $<0.0001$ & & & & \\
\hline$\geq 20$ & 105 & $9.0(6.0,14.0)$ & $6.1(4.2,8.8)$ & $<0.0001$ & & & & \\
\hline $\begin{array}{l}\text { Ki67 index } \\
\text { (MT=Yes) }\end{array}$ & & & & & & & $0.62(0.59,0.65)$ & $0.12(0.06,0.19)$ \\
\hline $0-9$ & 112 & $106.1(49.0, N R)$ & $1.2(0.8,1.8)$ & 0.504 & $0.16(-0.35,0.68)$ & 0.535 & & \\
\hline $10-19$ & 130 & $29.0(22.0,44.5)$ & $2.3(1.6,3.4)$ & $<0.0001$ & $-0.61(-1.65,0.42)$ & 0.246 & & \\
\hline$\geq 20$ & 239 & $19.7(14.0,23.0)$ & $3.4(2.4,4.8)$ & $<0.0001$ & $-2.72(-4.32,-1.11)$ & 0.001 & & \\
\hline $\begin{array}{l}\text { Resection } \\
\text { status } \\
\text { (MT= No) }\end{array}$ & & & & & & & $0.59(0.56,0.62)$ & $0.18(0.09,0.27)$ \\
\hline RO & 260 & $40.0(30.0,60.0)$ & 1 & Reference & & & & \\
\hline $\mathrm{RX}$ & 36 & $7.0(3.0,16.0)$ & $2.8(1.9,4.3)$ & $<0.0001$ & & & & \\
\hline $\mathrm{R} 1$ & 18 & $7.0(2.9,16.0)$ & $4.1(2.4,6.9)$ & $<0.0001$ & & & & \\
\hline $\begin{array}{l}\text { Resection } \\
\text { status } \\
\text { (MT=Yes) }\end{array}$ & & & & & & & $0.57(0.54,0.59)$ & $0.09(0.04,0.16)$ \\
\hline RO & 379 & $34.0(25.0,48.9)$ & $1.0(0.8,1.3)$ & 0.836 & $0.02(-0.20,0.25)$ & 0.837 & & \\
\hline $\mathrm{RX}$ & 65 & $22.0(13.0,29.0)$ & $1.8(1.3,2.5)$ & 0.001 & $-1.03(-2.20,0.15)$ & 0.087 & & \\
\hline $\mathrm{R} 1$ & 37 & $11.0(6.0,17.0)$ & $2.47(1.6,3.7)$ & $<0.0001$ & $-1.60(-3.83,0.62)$ & 0.158 & & \\
\hline $\begin{array}{l}\text { S-GRAS group } \\
(\mathrm{MT}=\mathrm{No})\end{array}$ & & & & & & & $0.72(0.69,0.76)$ & $0.30(0.22,0.38)$ \\
\hline $0-1$ & 98 & $199.8(199.8, N R)$ & 1 & Reference & & & & \\
\hline $2-3$ & 122 & $33.0(21.6,44.6)$ & $3.1(1.9,4.9)$ & $<0.0001$ & & & & \\
\hline $4-5$ & 76 & $7.0(5.0,10.0)$ & $9.3(5.8,14.9)$ & $<0.0001$ & & & & \\
\hline $6-9$ & 18 & $6.0(2.9,15.0)$ & $11.6(6.1,21.9)$ & $<0.0001$ & & & & \\
\hline $\begin{array}{l}\text { S-GRAS group } \\
(\mathrm{MT}=\text { Yes })\end{array}$ & & & & & & & $0.66(0.63,0.69)$ & $0.18(0.12,0.25)$ \\
\hline $0-1$ & 68 & $181.6(75.0, \mathrm{NR})$ & $1.0(0.5,1.8)$ & 0.976 & $-0.01(-0.63,0.61)$ & 0.975 & & \\
\hline $2-3$ & 238 & $34.0(25.0,48.0)$ & $2.6(1.7,4.1)$ & $<0.0001$ & $-0.49(-1.37,0.38)$ & 0.271 & & \\
\hline $4-5$ & 144 & $13.5(9.8,16.5)$ & $5.4(3.4,8.4)$ & $<0.0001$ & $-3.94(-6.93,-0.94)$ & 0.010 & & \\
\hline $6-9$ & 31 & $12.0(6.0,17.0)$ & $7.9(4.5,13.8)$ & $<0.0001$ & $-3.76(-10.42,2.89)$ & 0.268 & & \\
\hline
\end{tabular}

$\mathrm{HR}$, hazard ratio; NR, not reached (= median survival not reached, that is, percentage survival remained $>50 \%$ so this value and/or its $95 \%$ Cls cannot be computed).

60 months as well as the HR values, 95\% CI and $P$-values for PFS and DSS are reported in Fig. 3A, B, Table 1, and Supplementary Table 6. Briefly, clearer stratification was observed comparing S-GRAS 1 with 2 (DSS HR 2.1, PFS HR 1.9), 3 (DSS HR 3.6, PFS HR 3.3), 4 (DSS HR 5.7, PFS HR 5.1), and 5 (DSS HR 10.0, PFS HR 8.7). Patients with S-GRAS $0(4 \%)$ showed a reduced risk of progression or death compared to S-GRAS 1 (PFS HR 0.7, DSS HR 0.3), though this was not statistically significant. Individual S-GRAS scores 6-9 did not provide clear stratification. Notably, discrimination statistics for the individual S-GRAS scores showed the highest $\mathrm{C}$-index and $\mathrm{R}^{2}{ }_{\mathrm{D}}$ statistic (PFS: C-index $=0.73, \mathrm{R}_{\mathrm{D}}^{2}=0.30$, Table 1; DSS: C-index $=0.79$, $\mathrm{R}_{\mathrm{D}}^{2}=0.45$, respectively, Supplementary Table 6). 
To investigate the potential role of individual S-GRAS scores in guiding radiological surveillance, we assessed the percentage of ACC patients without disease progression or recurrence within the first 3 and 6 months after primary surgery. We observed the biggest differences in patients with S-GRAS 2, 3, 4, and 5 with an increasing frequency of disease progression in the first 6 months of surveillance (Fig. 3C and D).

\section{Prognostic performance of S-GRAS on recurrence- free survival}

Considering only patients with R0 status ( $n=648)$, ENSAT staging, Ki67 index, and symptoms - but not age - represented significant prognostic factors at univariable analysis. Overall, the discriminative performance of S-GRAS (C-index $=0.67)$ remained superior to ENSAT staging $(\mathrm{C}$-index $=0.62, P=0.001)$, age $(\mathrm{C}$-index $=0.52$,
Ki67 0-9\%, 10-19\%, and $\geq 20 \%$ groups (C-index $=0.65$, $P=0.052$ ). The corresponding $\mathrm{R}_{\mathrm{D}}^{2}$ figures were $0.23,0.11$, $0.01,0.08$, and 0.18 , respectively.

\section{Prognostic performance of S-GRAS and adjuvant mitotane}

We included 795 patients eligible for this analysis; 481 adjuvant mitotane-treated (60.5\%) and 314 untreated (39.5\%) (Supplementary Table 7). Mitotane-treated patients more frequently presented with symptoms (70\% vs $63 \%, P=0.034)$ and had higher Ki67 index ( $\geq 20$ in $50 \%$ vs $33 \%, P<0.0001)$.

\section{Recurrence-free survival}

S-GRAS had superior discriminatory performance compared to its individual components in both mitotanetreated and -untreated cohorts. The C-index and $\mathrm{R}^{2}{ }_{\mathrm{D}}$
A
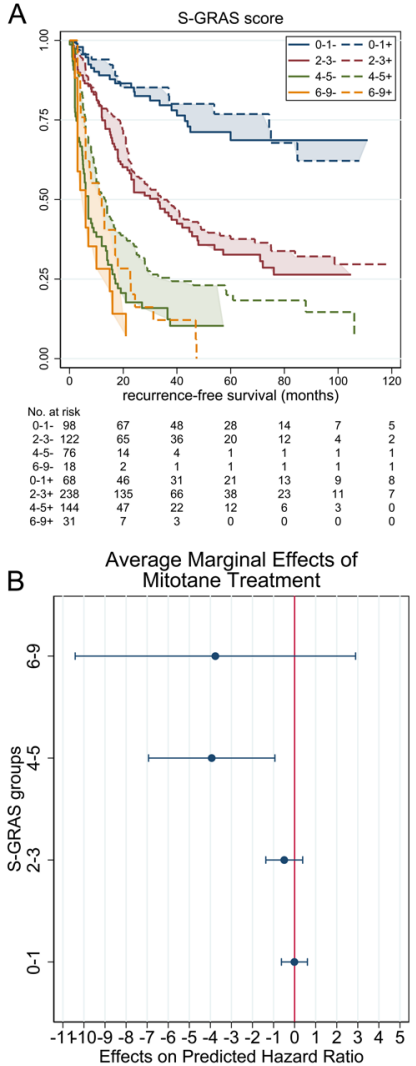
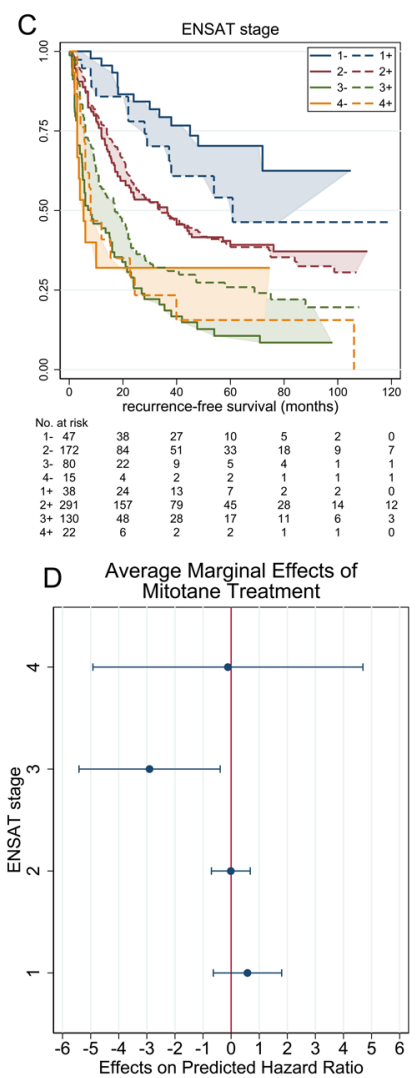

E
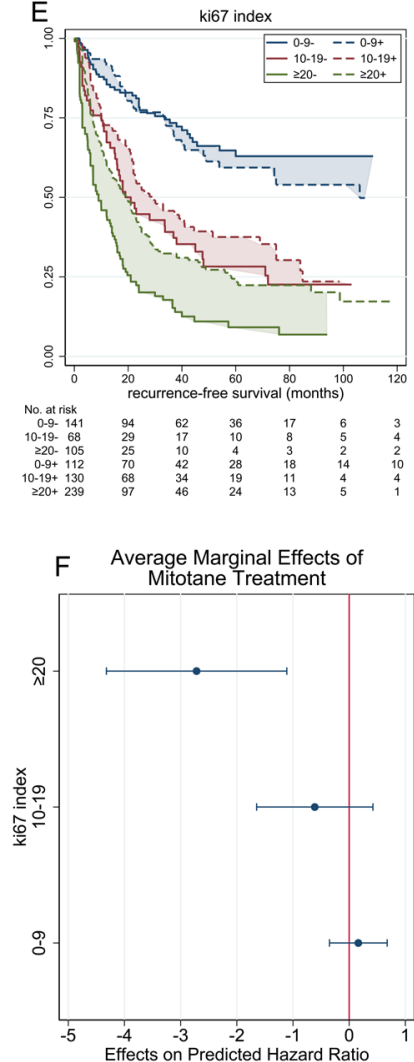
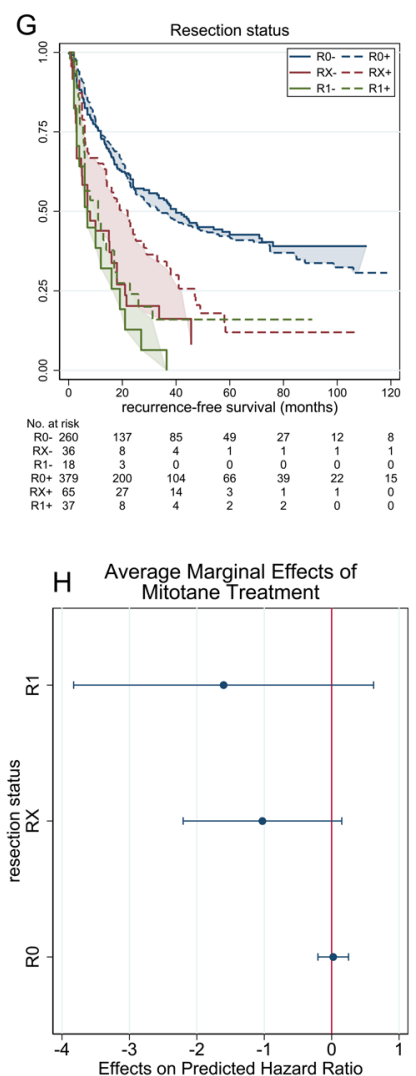

\section{Figure 4}

Kaplan-Meier (KM) curves depicting recurrence-free survival in patients untreated or treated with adjuvant mitotane; scatter plots showing the change in hazard ratio (marginal effects) as a result of treatment. According to (A and B) S-GRAS score groups, (C and D) ENSAT stage, (E and F) Ki67, and ( $G$ and H) resection status. ' - ' and ' + ' signs in the legends of the KM plots indicate no treatment and with treatment, respectively. 
statistics were higher for the S-GRAS groups compared to ENSAT stage, Ki67 index, and resection status (Table 2). Moreover, we compared the disease recurrence risk in treated and untreated patients in each S-GRAS group. Interestingly, there was a trend to reduction in the HR across the S-GRAS groups with mitotane treatment. This reduction was only statistically significant in S-GRAS 4-5 $(P=0.010)$, where HR fell from 9.3 (95\% CI 5.8-15.0) to 5.4 (95\% CI 3.4-8.4) (Fig. 4A, B and Table 2). Considering ENSAT staging, only the change at ENSAT stage 3 was statistically significant (from 7.1, 95\% CI 4.0-13.0, to 4.2, 95\% CI 2.3-7.5, $P=0.024$ ) (Fig. 4C, D and Table 2). Regarding Ki67 index, only patients with Ki67 $\geq 20 \%$ showed a statistically significant reduction in HR with mitotane (from $6.0,95 \%$ CI $4.2-8.8$, to 3.4 , 95\% CI $2.4-$ 4.7; $P=0.001$, Fig. 4E, F and Table 2). No changes in HR at each resection status were statistically significant (Fig. 4G, $\mathrm{H}$ and Table 2).

\section{Disease-specific survival}

The C-index and $\mathrm{R}_{\mathrm{D}}^{2}$ statistics were again higher for the S-GRAS groups compared to ENSAT stage, Ki67 index, and resection status. Supplementary Fig. 2 and Supplementary Table 8 summarises the marginal effects of mitotane treatment for disease-related death. Despite trending HR changes as a result of covariate change from untreated to treated with adjuvant mitotane, none were statistically significant.

\section{Discussion}

This is, to our knowledge, the largest multicentre study on comprehensive prognostic stratification in ACC patients based on clinical/histopathological characteristics, which enabled us to accurately assess the prognostic performance of the newly proposed S-GRAS score (19). Previous studies suggested promising prognostic biomarkers at the tumour level $(19,25,26,27$, 28 ). However, molecular analysis is not routine practice in ACC. Contrarily, the S-GRAS components are available as part of standard clinical practice for operated ACC patients.

We demonstrated superior prognostic discrimination for both DSS and PFS by the S-GRAS score compared to ENSAT staging (3) and Ki67 index (9), the current standard ACC prognostic tools (6). Moreover, the ten individual S-GRAS score values showed even better identification of further subgroups of patients with different clinical outcome. Hereby, it is worth mentioning that not all individual S-GRAS components carry the same weight with some (e.g. ENSAT stage and Ki67) having a stronger influence on PFS/DSS compared to others.

Few recent studies proposed a combination of clinical/histopathological characteristics to improve prognostication in ACC $(20,29)$ and the prognostic role of different combinations based on mathematical models has also been reported $(30,31,32,33,34)$. However, these studies included small cohorts of patients and/

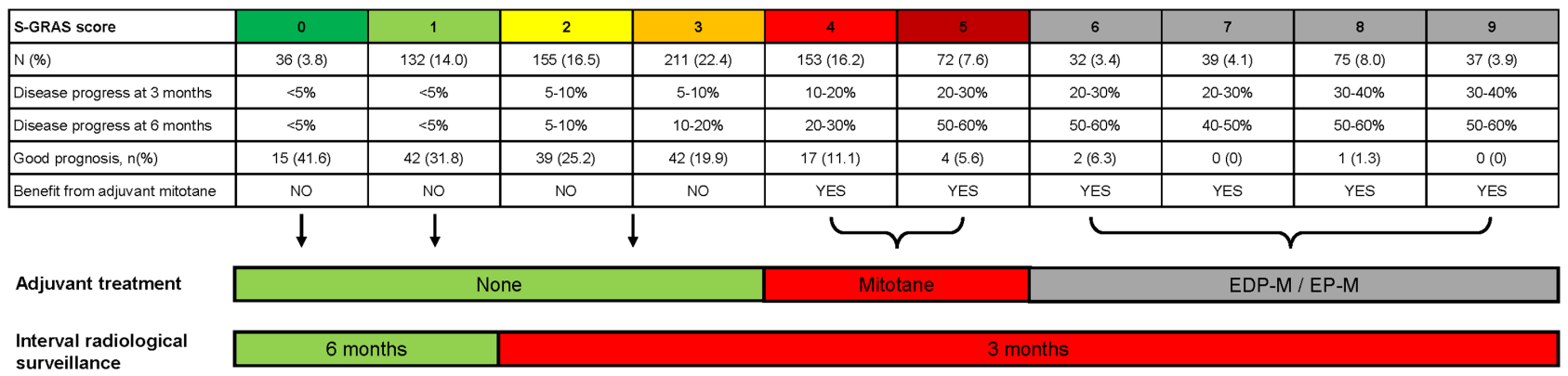

\section{Figure 5}

Proposal for the potential use of S-GRAS score categories in the clinical practice. Table showing frequency of individual S-GRAS scores, percentage of patients with disease progression at surveillance radiological imaging at 3 and 6 months after primary surgery, percentage of patients with favourable prognosis and potential benefit from adjuvant mitotane treatment according to the findings of the present paper. Patients were defined to have a favourable prognosis by progression-free survival $\geq 24$ months and disease-specific survival $\geq 60$ months (19). Suggestion for the potential use of S-GRAS categories in the clinical decisionmaking process: (A) adjuvant treatment with mitotane or cytotoxic drugs plus mitotane (i.e. EDP-M, Etoposide-DoxorubicineCisPlatin or EP-M, Etoposide-CisPlatin); (B) interval of radiological surveillance. 
or heterogeneous variables or outcomes and/or did not include a comparative statistics, Supplementary Table 9.

The modified version of ENSAT stage (mENSAT) (8) was not considered in the present study due to the selection bias that excluded non-operable patients. Future studies are therefore needed to investigate the added value of GRAS components to mENSAT classification in non-resectable disease.

Mitotane is the mainstay adjuvant therapy in ACC, which can carry significant toxicity. Mitotane use is based on retrospective and conflicting evidence $(4,5,21,35,36,37$, 38 ), and there are no reliable markers to predict treatment response (20). Currently, adjuvant mitotane is proposed for patients considered at high risk of recurrence (Ki67 index $\geq 10 \%$ and/or resection status RX-R1, and/or ENSAT stage 3 and 4) $(6,10)$. Results of the first prospective study on mitotane adjuvant in low-risk patients are still awaited (ADIUVO, ClinicalTrials.gov identifier 777244). Here, we firstly show that only patients with Ki67 index $\geq 20 \%$ and/ or ENSAT stage 3 had a significant longer RFS if treated with adjuvant mitotane - independently from the resection status. Moreover, S-GRAS seems to best stratify patients with different outcomes whether patients received mitotane or not, with S-GRAS score values $4-5$ being associated with longer RFS in mitotane-treated patients. Therefore, we hereby hypothesise that S-GRAS can be used also to stratify patients more likely to benefit from adjuvant mitotane.

We hereby propose a novel, improved management strategy for operated ACC patients, focusing on S-GRAS scoring, as compared to the current European guideline (Fig. 5 and Supplementary Table 10) (6). First, we suggest that S-GRAS $0-1$ patients (18\%) might be offered longer radiological surveillance intervals (e.g. every 6 instead of the 3 months recommended by current guidelines) $(6,10)$. This may reduce radiation exposure to patients with a low likelihood of recurrence. Second, patients with S-GRAS 0-3 seem less likely to benefit from adjuvant mitotane and could be saved from this treatment (avoiding unnecessary adverse effects). However, specifically in S-GRAS group $2-3$, the direct role of Ki67 on effects of adjuvant mitotane needs to be further validated. We also hypothesise that S-GRAS score 6-9 may better pre-select higher risk ACC patients suited for a more aggressive adjuvant approach such as cytotoxic chemotherapy. In fact, a very recent retrospective study suggests that patients with very high risk for recurrence benefit from adjuvant chemotherapy with a platinum-based therapy (39). Furthermore, this approach is currently under investigation in prospective trials (ADIUVO-2 trial, ClinicalTrials.gov identifier NCT03583710, and ACACIA trial, NCT03723941).
Limitations of this study comprise its retrospective nature and the non-centralised radiological and histopathological reporting. Like all prognostic studies, the indirect impact of additional adjuvant treatments (i.e. local radiotherapy) or therapeutic interventions (i.e. subsequent local therapies, cytotoxic drugs) on overall survival cannot be excluded. However, our study's major strengths, that is, the unique, well clinically annotated and vast patient cohort, standardised method of data extraction from ENSAT registry, and expert statistical analysis, allowed robust conclusions in this rare cancer.

In conclusion, our findings on a large cohort of ACC patients demonstrate that S-GRAS scoring can improve the management of ACC, personalising the frequency of radiological surveillance and rationalising the use of adjuvant mitotane after radical surgery. This S-GRAS-based strategy now requires validation in future prospective studies aimed to compare the prognostic role of S-GRAS (for predicting disease recurrences and response to mitotane treatment) before being implemented in clinical practice.

\section{Supplementary materials}

This is linked to the online version of the paper at https://doi.org/10.1530/ EJE-21-0510.

\section{Declaration of interest}

A J Sitch, W Arlt, and M. Fassnacht are on the editorial board of EJE. A J Sitch, W Arlt, and M Fassnacht were not involved in the review or editorial process for this paper, on which they are listed as an author.

\section{Funding}

This study was supported by the following funding sources: Deutsche Forschungsgemeinschaft (DFG) within the CRC/Transregio (314061271 TRR 205 to M F and M K), project FA-466/8-1 and RO-5435/3-1 (to M F, M $K$, and $C L R$, respectively), and project FA-466/4-2 (to $M F$ ), the Deutsche Krebshilfe (70113526 to M F and C L R), Fundacao de Amparo a Pesquisa do Estado de Sao Paulo (FAPESP) to M C B V F (2017/26345-5). This paper presents independent research supported by the NIHR Birmingham Biomedical Research Centre at the University Hospitals Birmingham NHS Foundation Trust and the University of Birmingham. The views expressed are those of the author(s) and not necessarily those of the NHS, the NIHR or the Department of Health and Social Care (S B, A S, J D, and W A).

\section{Acknowledgements}

The authors thank Miss Harriet Cook from the University of Birmingham (UK) and Mrs Michaela Haaf from the University of Wuerzburg (Germany) for their help with compiling the data.

\section{References}

1 Kebebew E, Reiff E, Duh QY, Clark OH \& McMillan A. Extent of disease at presentation and outcome for adrenocortical carcinoma: have we made progress? World Journal of Surgery 200630 872-878. (https://doi. org/10.1007/s00268-005-0329-x) 
2 Kerkhofs TMA, Verhoeven RHA, Van Der Zwan JM, Dieleman J, Kerstens MN, Links TP, Van de Poll-Franse LV \& Haak HR.

Adrenocortical carcinoma: a population-based study on incidence and survival in the Netherlands since 1993. European Journal of Cancer 2013 49 2579-2586. (https://doi.org/10.1016/j.ejca.2013.02.034)

3 Fassnacht M, Johanssen S, Quinkler M, Bucsky P, Willenberg HS, Beuschlein F, Terzolo M, Mueller HH, Hahner S, Allolio B et al. Limited prognostic value of the 2004 International Union Against Cancer staging classification for adrenocortical carcinoma: proposal for a revised TNM classification. Cancer 2009115 243-250. (https://doi. org/10.1002/cncr.24030)

4 Else T, Williams AR, Sabolch A, Jolly S, Miller BS \& Hammer GD. Adjuvant therapies and patient and tumor characteristics associated with survival of adult patients with adrenocortical carcinoma. Journal of Clinical Endocrinology and Metabolism 201499 455-461. (https://doi. org/10.1210/jc.2013-2856)

5 Calabrese A, Basile V, Puglisi S, Perotti P, Pia A, Saba L, Berchialla P, Porpiglia F, Veltri A, Volante $\mathrm{M}$ et al. Adjuvant mitotane therapy is beneficial in non-metastatic adrenocortical carcinoma at high risk of recurrence. European Journal of Endocrinology 2019180 387-396. (https://doi.org/10.1530/EJE-18-0923)

6 Fassnacht M, Dekkers OM, Else T, Baudin E, Berruti A, De Krijger RR, Haak HR, Mihai R, Assie G \& Terzolo M. European society of endocrinology clinical practice guidelines on the management of adrenocortical carcinoma in adults, in collaboration with the European Network for the study of adrenal tumors. European Journal of Endocrinology 2018179 G1-G46. (https://doi.org/10.1530/EJE-18-0608)

7 Lughezzani G, Sun M, Perrotte P, Jeldres C, Alasker A, Isbarn H, Budaus L, Shariat SF, Guazzoni G, Montorsi F et al. The European Network for the study of adrenal tumors staging system is prognostically superior to the international union against cancerstaging system: a North American validation. European Journal of Cancer 201046 713-719. (https://doi.org/10.1016/j.ejca.2009.12.007)

8 Libé R, Borget I, Ronchi CL, Zaggia B, Kroiss M, Kerkhofs T, Bertherat J, Volante M, Quinkler M, Chabre O et al. Prognostic factors in stage III-IV adrenocortical carcinomas (ACC): an European Network for the Study of Adrenal Tumor (ENSAT) study. Annals of Oncology 201526 2119-2125. (https://doi.org/10.1093/annonc/ mdv329)

9 Beuschlein F, Weigel J, Saeger W, Kroiss M, Wild V, Daffara F, Libe R, Ardito A, Al Ghuzlan A, Quinkler M et al. Major prognostic role of Ki67 in localized adrenocortical carcinoma after complete resection. Journal of Clinical Endocrinology and Metabolism 2015100 841-849. (https:// doi.org/10.1210/jc.2014-3182)

10 Fassnacht M, Assie G, Baudin E, Eisenhofer G, de la Fouchardiere C, Haak HR, de Krijger R, Porpiglia F, Terzolo M, Berruti A et al. Adrenocortical carcinomas and malignant phaeochromocytomas: ESMO-EURACAN clinical practice guidelines for diagnosis, treatment and follow-up. Annals of Oncology 202031 1476-1490. (https://doi. org/10.1016/j.annonc.2020.08.2099)

11 Else T, Kim AC, Sabolch A, Raymond VM, Kandathil A, Caoili EM, Jolly S, Miller BS, Giordano TJ \& Hammer GD. Adrenocortical carcinoma. Endocrine Reviews 201435 282-326. (https://doi. org/10.1210/er.2013-1029)

12 Erdogan I, Deutschbein T, Jurowich C, Kroiss M, Ronchi C, Quinkler M, Waldmann J, Willenberg HS, Beuschlein F, Fottner C et al. The role of surgery in the management of recurrent adrenocortical carcinoma. Journal of Clinical Endocrinology and Metabolism 201398 181-191. (https://doi.org/10.1210/jc.2012-2559)

13 Johanssen S, Hahner S, Saeger W, Quinkler M, Beuschlein F, Dralle H, Haaf M, Kroiss M, Jurowich C, Langer P et al. Deficits in the management of patients with adrenocortical carcinoma in Germany. Deutsches Arzteblatt International 2010107 885-891. (https://doi. org/10.3238/arztebl.2010.0885)

14 Abiven G, Coste J, Groussin L, Anract P, Tissier F, Legmann P, Dousset B, Bertagna X \& Bertherat J. Clinical and biological features in the prognosis of adrenocortical cancer: poor outcome of cortisolsecreting tumors in a series of 202 consecutive patients. Journal of Clinical Endocrinology and Metabolism 200691 2650-2655. (https://doi. org/10.1210/jc.2005-2730)

15 Berruti A, Fassnacht M, Haak H, Else T, Baudin E, Sperone P, Kroiss M, Kerkhofs T, Williams AR, Ardito A et al. Prognostic role of overt hypercortisolism in completely operated patients with adrenocortical cancer. European Urology 201465 832-838. (https://doi.org/10.1016/j. eururo.2013.11.006)

16 Vanbrabant T, Fassnacht M, Assie G \& Dekkers OM. Influence of hormonal functional status on survival in adrenocortical carcinoma: systematic review and meta-analysis. European Journal of Endocrinology 2018179 429-436. (https://doi.org/10.1530/EJE-18-0450)

17 Assié G, Antoni G, Tissier F, Caillou B, Abiven G, Gicquel C, Leboulleux S, Travagli JP, Dromain C, Bertagna X et al. Prognostic parameters of metastatic adrenocortical carcinoma. Journal of Clinical Endocrinology and Metabolism 200792 148-154. (https://doi. org/10.1210/jc.2006-0706)

18 Margonis GA, Kim Y, Tran TB, Postlewait LM, Maithel SK, Wang TS, Glenn JA, Hatzaras I, Shenoy R, Phay JE et al. Outcomes after resection of cortisol-secreting adrenocortical carcinoma. American Journal of Surgery 2016211 1106-1113. (https://doi.org/10.1016/j. amjsurg.2015.09.020)

19 Lippert J, Appenzeller S, Liang R, Sbiera S, Kircher S, Altieri B, Nanda I, Weigand I, Gehrig A, Steinhauer S et al. Targeted molecular analysis in adrenocortical carcinomas: a strategy toward improved personalized prognostication. Journal of Clinical Endocrinology and Metabolism 2018 103 4511-4523. (https://doi.org/10.1210/jc.2018-01348)

20 Bedrose S, Daher M, Altameemi L \& Habra MA. Adjuvant therapy in adrenocortical carcinoma: reflections and future directions. Cancers 202012 508. (https://doi.org/10.3390/cancers12020508)

21 Terzolo M, Angeli A, Fassnacht M, Daffara F, Tauchmanova L, Conton PA, Rossetto R, Buci L, Sperone P, Grossrubatscher E et al. Adjuvant mitotane treatment for adrenocortical carcinoma. New England Journal of Medicine 2007356 2372-2380. (https://doi. org/10.1056/NEJMoa063360)

22 Jinks RC, Royston P \& Parmar MK. Discrimination-based sample size calculations for multivariable prognostic models for time-to-event data. BMC Medical Research Methodology 201515 82. (https://doi. org/10.1186/s12874-015-0078-y)

23 Harrell FE, Califf RM, Pryor DB, Lee KL \& Rosati RA. Evaluating the yield of medical tests. JAMA 1982247 2543-2546. (https://doi. org/10.1001/jama.247.18.2543)

24 Newson RB. Comparing the predictive powers of survival models using Harrell's C or Somers' D. STATA Journal: Promoting Communications on Statistics and Stata 201010 339-358. (https://doi.org/10.1177/15368 67X1001000303)

25 De Reyniès A, Assié G, Rickman DS, Tissier F, Groussin L, René-Corail F, Dousset B, Bertagna X, Clauser E \& Bertherat J. Gene expression profiling reveals a new classification of adrenocortical tumors and identifies molecular predictors of malignancy and survival. Journal of Clinical Oncology 200927 1108-1115. (https://doi.org/10.1200/ JCO.2008.18.5678)

26 Assié G, Letouzé E, Fassnacht M, Jouinot A, Luscap W, Barreau O, Omeiri H, Rodriguez S, Perlemoine K, René-Corail F et al. Integrated genomic characterization of adrenocortical carcinoma. Nature Genetics 201446 607-612. (https://doi.org/10.1038/ng.2953)

27 Zheng S, Cherniack AD, Dewal N, Moffitt RA, Danilova L, Murray BA, Lerario AM, Else T, Knijnenburg TA, Ciriello G et al. Comprehensive pan-genomic characterization of adrenocortical carcinoma. Cancer Cell 201629 723-736. (https://doi.org/10.1016/j.ccell.2016.04.002)

28 Jouinot A, Assie G, Libe R, Fassnacht M, Papathomas T, Barreau O, de la Villeon B, Faillot S, Hamzaoui N, Neou M et al. DNA methylation is an independent prognostic marker of survival in adrenocortical cancer. Journal of Clinical Endocrinology and Metabolism 2017 102 923-932. (https://doi.org/10.1210/jc.2016-3205) 
29 Liang J, Liu Z, Zhou L, Tang Y, Zhou C, Wu K, Zhang F, Zhang F, Wei X, Lu Y et al. The clinical utility of 'GRAS' parameters in stage I-III adrenocortical carcinomas: long-term data from a high-volume institution. Endocrine 202067 449-456. (https://doi.org/10.1007/ s12020-019-02141-2)

30 Kim Y, Margonis GA, Prescott JD, Tran TB, Postlewait LM, Maithel SK, Wang TS, Evans DB, Hatzaras I, Shenoy R et al. Nomograms to predict recurrence-free and overall survival after curative resection of adrenocortical carcinoma. JAMA Surgery 2016151 365-373. (https:// doi.org/10.1001/jamasurg.2015.4516)

31 Li Y, Bian X, Ouyang J, Wei S, He M \& Luo Z. Nomograms to predict overall survival and cancer-specific survival in patients with adrenocortical carcinoma. Cancer Management and Research 201810 6949-6959. (https://doi.org/10.2147/CMAR.S187169)

32 Kong J, Zheng J, Cai J, Wu S, Diao X, Xie W, Chen X, Liao C, Yu H, Fan X et al. A nomogram for individualized estimation of survival among adult patients with adrenocortical carcinoma after surgery: a retrospective analysis and multicenter validation study. Cancer Communications 2019 39 80. (https://doi.org/10.1186/s40880-019-0426-0)

33 Ettaieb MHT, van Kuijk SMJ, de Wit-Pastoors A, Feelders RA, Corssmit EPM, Eekhoff EMW, van der Valk P, Timmers HJLM, Kerstens MN, Klümpen HJ et al. Development and internal validation of a multivariable prediction model for adrenocortical-carcinomaspecific mortality. Cancers 202012 1-16. (https://doi.org/10.3390/ cancers12092720)

34 Baechle JJ, Marincola Smith P, Solórzano CC, Tran TB, Postlewait LM, Maithel SK, Prescott J, Pawlik T, Wang TS, Glenn J et al. Cumulative GRAS score as a predictor of survival after resection for adrenocortical carcinoma: analysis from the U.S. adrenocortical carcinoma database. Annals of Surgical Oncology 202128 6551-6561. (https://doi. org/10.1245/s10434-020-09562-8)

35 Fassnacht M, Johanssen S, Fenske W, Weismann D, Agha A, Beuschlein F, Führer D, Jurowich C, Quinkler M, Petersenn S et al. Improved survival in patients with Stage II adrenocortical carcinoma followed up prospectively by specialized centers. Journal of Clinical Endocrinology and Metabolism 201095 4925-4932. (https://doi. org/10.1210/jc.2010-0803)

36 Berruti A, Grisanti S, Pulzer A, Claps M, Daffara F, Loli P, Mannelli M, Boscaro M, Arvat E, Tiberio G et al. Long-term outcomes of adjuvant mitotane therapy in patients with radically resected adrenocortical carcinoma. Journal of Clinical Endocrinology and Metabolism 2017102 1358-1365. (https://doi.org/10.1210/jc.2016-2894)

37 Postlewait LM, Ethun CG, Tran TB, Prescott JD, Pawlik TM, Wang TS, Glenn J, Hatzaras I, Shenoy R, Phay JE et al. Outcomes of adjuvant mitotane after resection of adrenocortical carcinoma: a 13-institution study by the US Adrenocortical Carcinoma Group. Journal of the American College of Surgeons 2016222 480-490. (https://doi. org/10.1016/j.jamcollsurg.2015.12.013)

38 Grubbs EG, Callender GG, Xing Y, Perrier ND, Evans DB, Phan AT \& Lee JE. Recurrence of adrenal cortical carcinoma following resection: surgery alone can achieve results equal to surgery plus mitotane. Annals of Surgical Oncology 201017 263-270. (https://doi.org/10.1245/ s10434-009-0716-x)

39 Kimpel O, Bedrose S, Megerle F, Berruti A, Terzolo M, Kroiss M, Mai K, Dekkers OM, Habra MA \& Fassnacht M. Adjuvant platinum-based chemotherapy in radically resected adrenocortical carcinoma: a cohort study. British Journal of Cancer 2021125 1233-1238. (https:// doi.org/10.1038/s41416-021-01513-8)

Received 14 May 2021

Revised version received 6 October 2021

Accepted 27 October 2021 